\title{
Technical note: Methods for interim prediction of single-step breeding values for young animals
}

\author{
E. C. G. Pimentel, ${ }^{*}$ C. Edel, R. Emmerling, and K.-U. Götz \\ Institute of Animal Breeding, Bavarian State Research Center for Agriculture, 85586 Grub, Germany
}

\section{ABSTRACT}

Single-step genomic evaluations have the advantage of simultaneously combining all pedigree, phenotypic, and genotypic information available. However, systems with a large number of genotyped animals have some computational challenges. In many genomic breeding programs, genomic predictions of young animals should become available for selection decisions in the shortest time possible, which requires either a very effective estimation or an approximation with negligible loss in accuracy. We investigated different procedures for predicting breeding values of young genotyped animals without setting up the full single-step system augmented for the additional genotypes. Methods were based on transmitting the information from single-step breeding values of genotyped animals that took part in the previous full run to young animals, either through genomic relationships or through a marker-based model. The different procedures were tested on real data from the April 2017 run of the German-Austrian official genomic evaluation for Fleckvieh. The data set included 62,559 genotyped animals and was used to run single-step evaluations for 23 conformation traits. A further data set comprising 1,768 young animals was used for interim prediction and we called it the validation set. The reference values for validation were the predicted breeding values of the young animals from a full single-step run containing the genotypes of all 64,327 animals. Correlations between the approximated predictions and those from the full single-step run also containing genotypes from young animals averaged 0.9932 for the best method (from 0.990 to 0.995 across traits). In conclusion, prediction of single-step breeding values for young animals can be well approximated using systems of size equal to the number of markers.

Key words: genomic breeding value, genotype, indirect prediction, SNP effect

Received August 24, 2018.

Accepted December 18, 2018.

*Corresponding author: Eduardo.Pimentel@LfL.bayern.de

\section{Technical Note}

Single-step genomic evaluations have the advantage of simultaneously combining all pedigree, phenotypic, and genotypic information available in a single system of equations (Legarra et al., 2009; Christensen and Lund, 2010). This unified approach negates the need to compute some aggregated phenotype such as daughter yield deviations or deregressed proofs. It also makes it unnecessary to subsequently blend direct genomic values (DGV) with conventionally estimated breeding values to obtain final genomic breeding values (GEBV). Furthermore, single-step models may properly account for the genomic preselection that bias conventional evaluations in multi-step genomic systems (Patry and Ducrocq, 2011). However, single-step systems with a large number of genotyped animals are computationally demanding. In many genomic breeding programs, genomic predictions of young animals should become available for selection decisions in the shortest time possible, which requires either a very effective estimation or an approximation with negligible loss in accuracy. Wiggans et al. (2015) developed a method for rapid delivery of preliminary GEBV of young animals in the time between monthly evaluations in a multi-step genomic system. They highlighted the importance of this rapid evaluation in enabling breeders to make early culling decisions. Lourenco et al. (2015) presented an indirect method for predicting GEBV of young animals to be used for interim evaluations in a single-step system. Their approach was based on the transmission of DGV from reference to young animals and a weighted combination of these DGV with the parent average (PA). When the reference population was large, they found no major advantage of combining DGV with PA. In this study, we investigated further alternative procedures for predicting breeding values of young genotyped animals without setting up the full single-step system augmented for the additional genotypes.

The different procedures were tested on real data from the April 2017 run of the German-Austrian official genomic evaluation for Fleckvieh. Routine evaluations 
are based on the Illumina Bovine SNP50 BeadChip (Illumina Inc., San Diego, CA). After the usual edits, 41,276 markers remained for subsequent analyses. Detailed descriptions of the criteria used for marker editing and animal checks can be found in Edel et al. (2011). The final data set contained 62,559 genotyped animals and was used to run single-step evaluations for 23 conformation traits. Another data set comprising 1,768 young animals was used for interim prediction; we called it the validation set. The reference values for validation were the predicted breeding values of the young animals from a full single-step run, including the genotypes of all 64,327 animals.

Phenotypic data, pedigree, and fixed and random effects included in the models of all single-step analyses were the same as in the animal models used in the official conventional evaluations for conformation traits. The general model equation is

$$
\mathbf{y}=\mathbf{X b}+\mathbf{W h}+\mathbf{Z Q} \mathbf{c}+\mathbf{Z u}+\varepsilon,
$$

where $\mathbf{y}, \mathbf{b}, \mathbf{h}, \mathbf{c}, \mathbf{u}$, and $\varepsilon$ are the vectors of observations, fixed effects, random herd $\times$ year effects, random genetic group effects, random animal additive genetic effects, and random residuals, respectively. Incidence matrices $\mathbf{X}, \mathbf{W}$, and $\mathbf{Z}$ relate observations to fixed and random effects. Fixed effects consisted of an overall mean and the effects of herd, year $\times$ season, classifier $\times$ year, calving age, lactation stage, and parity. For udder traits, a fixed effect of time from last milking is also included. Matrix $\mathbf{Q}$ contains the fractions of each group effect contributing to the breeding value (a) of the animal, so that

$$
\hat{\mathbf{a}}=\hat{\mathbf{u}}+\mathbf{Q} \hat{\mathbf{c}}
$$

The number of animals in the pedigree was $3,172,636$ and the number of observations ranged from 690,654 to $1,395,262$ across the 23 conformation traits. Unknown parents were assigned to genetic groups. To avoid the transformation problems described in Misztal et al. (2013), genetic groups were explicitly modeled as covariates containing the contributions of the genetic groups to the animals with records. These covariates are the columns of the matrix $\mathbf{Q}$ (Westell et al., 1988). Genetic group contributions were calculated using the program RelaX2 version 1.75 update 12/2016 (Strandén and Vuori, 2006). Final GEBV of an animal was calculated as the sum of the random animal effect and the solutions for the group effects weighted by the contributions of each group to the animal. All single-step analyses were done using the software package MiX99
Release XI/2016 version 16.1108 (MiX99 Development Team, 2016) for solving the mixed model equations. These single-step analyses required the calculation of the block of the inverse of the covariance matrix $\mathbf{H}$ corresponding to the genotyped animals $\left[\mathbf{G}^{-1}-\mathbf{A}_{22}^{-1}\right]$, which was given as input to MiX99. Matrix $\mathbf{A}_{22}$ is Wright's numerator relationship matrix among the genotyped animals, and $\mathbf{G}$ is the genomic relationship matrix, which was combined with $20 \%$ numerator relationships (i.e., $\mathbf{G}=0.8 \mathbf{G}_{0}+0.2 \mathbf{A}_{22}$ ) to account for a proportion of the additive genetic variance that is not explained by the markers (Christensen and Lund, 2010). Henderson (1985) showed that adding the covariance matrices of 2 random factors in the model can be done to estimate the sum of their effects. He also showed how estimates for the individual factors can be calculated afterward from the estimate of the sum.

Matrix $\mathbf{G}_{0}$ was computed following the first method described by VanRaden (2008):

$$
\mathbf{G}_{0}=\mathbf{M M}^{\prime}\left[2 \sum_{i=1}^{m} p_{i}\left(1-p_{i}\right)\right]^{-1},
$$

where $m$ is the number of markers; $p_{i}$ is the base reference allele frequency at locus $i$; and $\mathbf{M}$ is the $(n \times m)$ matrix of centered genotype coefficients, with $n$ equal to the number of animals. Matrix $\mathbf{M}$ was calculated as $\mathbf{M}=\mathbf{Z}-\mathbf{P}$, where $\mathbf{Z}$ is the matrix of reference allele counts (coded as 0,1 , or 2 ) and $\mathbf{P}$ is a matrix of which the $i$ th column is $2 p_{i}$. Estimates of base allele frequencies were obtained using the method proposed by Gengler et al. (2007).

Three methods were used to predict the breeding values of the 1,768 young animals. The reference method for comparison was the full single-step model, in which the genotypes of all 64,327 animals were included in the calculation of the full genomic relationship matrix $\mathbf{G}_{f}$. The 2 alternative methods were based on the transmission of the information from single-step breeding values of the 62,559 genotyped animals that took part in the April 2017 run to the 1,768 young animals, either through genomic relationships (the $\mathrm{G}$ model) or through a marker based regression (the SNP model). In the $\mathrm{G}$ model, predicted values of young animals were calculated as

$$
\hat{\mathbf{a}}_{y}=\mathbf{C G}^{-1} \hat{\mathbf{a}}_{2},
$$

where $\hat{\mathbf{a}}_{2}$ is the $(62,559 \times 1)$ vector of single-step breeding values of the genotyped animals from the April 
2017 run; $\mathbf{G}^{-1}$ is the inverse of the $(62,559 \times 62,559)$ genomic relationship matrix among the genotyped animals from the April $2017 \mathrm{run}$; $\mathbf{C}$ is the $(1,768 \times 62,559)$ matrix of genomic relationships between the young animals and the genotyped animals from the April 2017 run; and $\hat{\mathbf{a}}_{y}$ is the $(1,768 \times 1)$ vector of single-step breeding values of the young animals.

Both matrices $\mathbf{G}^{-1}$ and $\mathbf{C}$ were combined with $20 \%$ numerator relationships, as for the full genomic relationship matrix $\mathbf{G}_{f}$ with 64,327 animals that was used in the full model. This method is similar to the method proposed by VanRaden (2008) for application in 2-step GBLUP models, and by Wang et al. (2012) and Lourenco et al. (2015) for single-step models. It is also similar to the way that genomic components could be separated from polygenic components in $\hat{\mathbf{a}}_{2}$, as shown by Henderson (1985). This could be achieved by leaving the $20 \%$ numerator relationships out of $\mathbf{C}$ (for the genomic part) or replacing $\mathbf{C}$ by a numerator relationships matrix (for the polygenic part). If the number of animals were large, a disadvantage of this method would be the need for setting up and inverting $\mathbf{G}$.

When the number of animals is much larger than the number of SNP, a marker-based model can be a good alternative to the G model. In the SNP model, allele substitution effects (i.e., SNP effects) were estimated using data from the April 2017 run and then used to predict the breeding values of young animals. The SNP effects were estimated by solving the following mixed model equations:

$$
\begin{gathered}
{\left[\begin{array}{cc}
\mathbf{1}^{\prime} \mathbf{1} & \mathbf{1}^{\prime} \mathbf{M} \\
\mathbf{M}^{\prime} \mathbf{1} & \mathbf{M}^{\prime} \mathbf{M}+\mathbf{I} k
\end{array}\right]\left[\begin{array}{c}
\hat{\mu} \\
\hat{\mathbf{g}}
\end{array}\right]=\left[\begin{array}{c}
\mathbf{1}^{\prime} \hat{\mathbf{a}}_{2} \\
\mathbf{M}^{\prime} \hat{\mathbf{a}}_{2}
\end{array}\right]} \\
\text { with } k=\frac{\sigma_{e}^{2}}{\sigma_{g}^{2}},
\end{gathered}
$$

where $\mathbf{1}$ is a $(62,559 \times 1)$ vector of ones; $\mathbf{M}$ is the $(62,559 \times m)$ centered genotype matrix of animals that took part in the April run; $\mathbf{I}$ is an identity matrix of order $m ; \hat{\mu}$ is the estimated overall mean; $\hat{\mathbf{g}}$ is the $(m \times$ 1) vector of estimated SNP effects; $\sigma_{e}^{2}$ is the residual variance; and $\sigma_{g}^{2}$ is the variance of allele substitution effects.

As described above, $\hat{\mathbf{a}}_{2}$ is the vector of single-step breeding values of genotyped animals from the April 2017 run, in which the proportion of additive genetic variance $\left(\sigma_{a}^{2}\right)$ explained by the markers was assumed to be $80 \%$ (i.e., $\mathbf{G}=0.8 \mathbf{G}_{0}+0.2 \mathbf{A}_{22}$ ). Therefore, the ratio $k$ was calculated assuming a "marker heritability" of 0.80 :

$$
\begin{gathered}
k=\frac{\sigma_{e}^{2}}{\sigma_{g}^{2}}=\frac{0.2 \sigma_{a}^{2}}{0.8 \sigma_{a}^{2} \gamma}, \\
\gamma=\frac{1}{\sum 2 p_{i}\left(1-p_{i}\right)}, \\
k=0.25 \sum 2 p_{i}\left(1-p_{i}\right) .
\end{gathered}
$$

The residuals from this SNP effect estimation model are estimated as

$$
\hat{\mathbf{e}}=\hat{\mathbf{a}}_{2}-(\hat{\mu}+\mathbf{M} \hat{\mathbf{g}}) .
$$

This model assumed a residual variance equal to $0.2 \sigma_{a}^{2}$. Therefore, these residuals contain the $20 \%$ polygenic variance not explained by the markers. Following this assumption, the residual polygenic term $\left(\hat{\mathbf{e}}_{y}\right)$ can be transmitted to the young animals through the following regression:

$$
\hat{\mathbf{e}}_{y}=\mathbf{A}_{y 2} \mathbf{A}_{22}^{-1} \hat{\mathbf{e}},
$$

where $\mathbf{A}_{22}^{-1}$ is the inverse of the $(62,559 \times 62,559) \mathrm{nu}-$ merator relationship matrix among the genotyped animals from the April 2017 run; and $\mathbf{A}_{y 2}$ is the $(1,768 \times$ $62,559)$ matrix of numerator relationships between the young animals and the genotyped animals from the April 2017 run.

The single-step breeding values of the young animals are finally calculated as the sum of marker effects and the residual polygenic term:

$$
\hat{\mathbf{a}}_{y}=\left(\hat{\mu}+\mathbf{M}_{y} \hat{\mathbf{g}}\right)+\hat{\mathbf{e}}_{y},
$$

where $\mathbf{M}_{y}$ is the $(1,768 \times m)$ centered genotype matrix of the young animals.

An alternative to avoid the computation of $\mathbf{A}_{y 2} \mathbf{A}_{22}^{-1}$ is to use the result of Fernando et al. (2014) and calculate the residual polygenic term $\hat{\mathbf{e}}_{y}$ as the solution to the following set of equations:

$$
\mathbf{A}^{y y} \hat{\mathbf{e}}_{y}=-\mathbf{A}^{y 2} \hat{\mathbf{e}}
$$

where $\mathbf{A}^{y y}$ and $\mathbf{A}^{y 2}$ are the corresponding blocks of $\mathbf{A}^{-1}$. These matrices are very sparse and can be easily computed, which makes this a cost-effective alternative. Another alternative to avoid computing $\mathbf{A}_{y 2} \mathbf{A}_{22}^{-1}$ or solving the above sparse system would be to approximate 
Table 1. Correlations between genomic breeding values (GEBV) from the full single-step model and from the alternative prediction models for young animals

\begin{tabular}{lccc}
\hline Trait & G model & SNP model & SNP model PLUS \\
\hline Rump angle & 0.990 & 0.984 & 0.993 \\
Rump length & 0.994 & 0.992 & 0.994 \\
Muscularity & 0.985 & 0.974 & 0.991 \\
Udder depth & 0.995 & 0.993 & 0.994 \\
Udder & 0.991 & 0.987 & 0.992 \\
Fetlock & 0.992 & 0.989 & 0.993 \\
Feet and legs & 0.995 & 0.993 & 0.994 \\
Width & 0.994 & 0.992 & 0.994 \\
Height at cross & 0.991 & 0.989 & 0.993 \\
Front teat placement & 0.995 & 0.993 & 0.994 \\
Frame & 0.991 & 0.989 & 0.992 \\
Udder cleanliness & 0.992 & 0.989 & 0.991 \\
Rump depth & 0.994 & 0.991 & 0.993 \\
Rear udder length & 0.992 & 0.989 & 0.990 \\
Hocks & 0.991 & 0.987 & 0.993 \\
Hock angularity & 0.995 & 0.993 & 0.995 \\
Teat thickness & 0.994 & 0.992 & 0.995 \\
Teat length & 0.993 & 0.991 & 0.994 \\
Teat placement & 0.994 & 0.992 & 0.994 \\
Foot angle & 0.992 & 0.988 & 0.994 \\
Fore udder length & 0.994 & 0.990 & 0.993 \\
Fore udder attachment & 0.994 & 0.991 & 0.994 \\
Suspensory ligament & 0.995 & 0.992 & 0.994 \\
Mean & 0.9927 & 0.9896 & 0.9932 \\
\hline IG & & &
\end{tabular}

${ }^{1} \mathrm{G}$ model: interim solutions for young bulls by explicitly setting up the necessary genomic relationship matrices and inverting the G matrix. Polygenic residual component is fully considered. No intermediate SNP effect estimation. ${ }^{2}$ SNP model: interim solutions for young bulls via SNP effect solutions. G matrix is neither set up nor inverted. Polygenic residual component is considered by approximation.

${ }^{3}$ SNP model PLUS: same as SNP model, plus additional adjustments (see text).

this residual polygenic term, $\hat{\mathbf{e}}_{y}$, with PA or 3 sire PA calculated from the residuals in ê. The 3 sire PA is calculated when the contribution from the dam is missing; that is, when the dam is not genotyped. Then, the PA is calculated taking into account the sire and the dam's sire and maternal grandsire.

Correlations between the approximated predictions and those from the full single-step run that also contain genotypes from young animals are presented in Table 1. Results from the SNP model were obtained using the term $\mathbf{A}_{y 2} \mathbf{A}_{22}^{-1}$ to calculate $\hat{\mathbf{e}}_{y}$. Correlations averaged 0.9927 for the $\mathrm{G}$ model (from 0.985 to 0.995 across traits) and 0.9896 for the SNP model (from 0.974 to 0.993 across traits). Correlations between solutions from the $\mathrm{G}$ model and SNP model averaged 0.9959 (from 0.992 to 0.998 across traits). The $\mathrm{G}$ model is expected to perform better than the SNP model because in the $\mathrm{G}$ model, the $20 \%$ polygenic component is considered in exactly the same way in the calculations of matrices $\mathbf{G}$ and $\mathbf{C}$ as in the calculation of $\mathbf{G}_{f}$. As mentioned above, the $\mathrm{G}$ model could also offer a more elegant way of separating the genomic and polygenic components but at the cost of computing $\mathbf{G}^{-1}$. In contrast, in the SNP model, the residual vector ê not only contains the $20 \%$ polygenic component but also some additional estimation error, so that the transmission of the polygenic information to the young animals is not completely precise. This difference also becomes apparent if regarding both models in the context of genomic BLUP: the G model would be exact and the SNP model would be approximate. Of course, in the context of single step, they are both approximate.

Lourenco et al. (2015) applied an indirect method similar to the $\mathrm{G}$ model to approximate GEBV for 3 growth traits in beef cattle. They reported correlations of 0.97 between GEBV from the full single-step model and GEBV from their indirect prediction method when the number of genotyped animals in the previous run was 33,162 . We had almost twice as many genotyped animals in the April 2017 run, which may partly explain the higher correlations observed. Lourenco et al. (2018) mentioned that a problem with their indirect prediction was a mean shift in the indirectly predicted GEBV. They suggested 3 forms of adjustments to finetune the indirect predictions and reported that they were unbiased after the corrections. One of the adjustments was to add the mean GEBV of the genotyped animals from the previous run to the indirect predictions. We also observed a mean shift in the GEBV from the G model. Average GEBV from the G model, SNP model, and full model for each trait are presented in 
Table 2. Average genomic breeding values (GEBV) from the full single-step model and from the alternative prediction models for young animals

\begin{tabular}{|c|c|c|c|c|}
\hline Trait & $\begin{array}{c}\text { Full } \\
\text { model }^{1}\end{array}$ & G model ${ }^{2}$ & $\begin{array}{c}\text { SNP } \\
\text { model }^{3}\end{array}$ & $\begin{array}{c}\text { SNP } \\
\text { model PLUS }\end{array}$ \\
\hline Rump angle & 102.30 & 103.90 & 102.38 & 102.32 \\
\hline Rump length & 105.06 & 106.15 & 105.02 & 105.00 \\
\hline Muscularity & 99.78 & 103.41 & 99.98 & 99.87 \\
\hline Udder depth & 102.18 & 102.96 & 102.12 & 102.12 \\
\hline Udder & 110.36 & 117.57 & 110.12 & 110.17 \\
\hline Fetlock & 103.99 & 106.94 & 103.96 & 103.92 \\
\hline Feet and legs & 106.30 & 108.98 & 106.09 & 106.05 \\
\hline Width & 101.93 & 101.99 & 101.91 & 101.90 \\
\hline Height at cross & 103.67 & 107.62 & 103.64 & 103.59 \\
\hline Front teat placement & 106.96 & 113.53 & 106.63 & 106.75 \\
\hline Frame & 103.86 & 106.49 & 103.62 & 103.60 \\
\hline Udder cleanliness & 102.90 & 108.53 & 102.87 & 102.87 \\
\hline Rump depth & 103.83 & 107.46 & 103.76 & 103.73 \\
\hline Rear udder length & 106.73 & 113.94 & 106.58 & 106.71 \\
\hline Hocks & 100.83 & 97.75 & 100.66 & 100.68 \\
\hline Hock angularity & 98.90 & 99.14 & 98.92 & 98.93 \\
\hline Teat thickness & 99.63 & 98.43 & 99.62 & 99.61 \\
\hline Teat length & 97.51 & 93.99 & 97.56 & 97.57 \\
\hline Teat placement & 106.20 & 110.81 & 106.01 & 106.05 \\
\hline Foot angle & 105.22 & 112.10 & 105.26 & 105.22 \\
\hline Fore udder length & 109.52 & 116.19 & 109.31 & 109.35 \\
\hline Fore udder attachment & 105.87 & 108.56 & 105.70 & 105.73 \\
\hline Suspensory ligament & 104.13 & 108.40 & 103.92 & 103.96 \\
\hline
\end{tabular}

${ }^{1}$ Full model: full single-step model including young bulls.

${ }^{2} \mathrm{G}$ model: interim solutions for young bulls by explicitly setting up the necessary genomic relationship matrices and inverting the G matrix. Polygenic residual component is fully considered. No intermediate SNP effect estimation.

${ }^{3} \mathrm{SNP}$ model: interim solutions for young bulls via SNP effect solutions. G matrix is neither set up nor inverted. Polygenic residual component is considered by approximation.

${ }^{4}$ SNP model PLUS: same as SNP model, plus additional adjustments (see text).

Table 2. There was no mean shift problem with the GEBV from the SNP model because $\hat{\mu}$ was simultaneously estimated when solving the mixed model equations for SNP effects. Solutions for the random SNP effects have BLUP and the solution for the fixed effect $(\hat{\mu})$ has best linear unbiased estimator properties, so there is no need to adjust the mean afterward.

It is clear that both the G model and the SNP model are approximation methods because the information from the April 2017 run is not transmitted to the young animals through the matrix $\mathbf{H}$, but through the matrices $\mathbf{G}$ and $\mathbf{C}$ or through the genotype matrices $\mathbf{M}$ and $\mathbf{M}_{y}$. Furthermore, in a single-step system, each genotype of a young animal that is included in the evaluation modifies the covariance matrix $\mathbf{H}$ and influences its own predicted breeding value and those of other animals (Shabalina et al., 2017). Therefore, any attempt to predict the breeding value of a young animal without setting up the complete system can only be approximate, and a correlation of 1 could not be achieved even if all the information from the full matrix $\mathbf{H}$ of the April 2017 run could be transmitted to the young animals.
In a closer inspection of the results from the SNP model, we looked at the correlations for different groups of young animals, specifically with respect to whether their parents took part in the April 2017 run and, if yes, whether they contributed to the evaluation with phenotypes or genotypes. Insights from this inspection can be summarized as follows:

- Sires of young animals are usually genotyped and have taken part in the previous run, so the information from the male side can be well transmitted to the young animals through the SNP effects.

- If the dam of a young animal did not contribute phenotypic information to the previous evaluation, then there will be no dam contribution to the young animal's breeding value in either the SNP model or in the full model.

- If the dam had both a phenotype and a genotype in the previous run, then her contribution to the breeding value of the young animal is also well transmitted through the SNP effects.

- If the dam had a phenotype but no genotype in the previous run, then her contribution to the 
breeding value of the young animal is only partly transmitted through the contribution of the maternal grandsire in the SNP model. The dam's Mendelian sampling information is not contained in the vector $\hat{\mathbf{a}}_{2}$ and thus cannot be transmitted to the young animal.

- If the dam is unknown, she is replaced by a genetic group in the full model. The contribution of this genetic group to the breeding value of the young animal is also not contained in the vector $\hat{\mathbf{a}}_{2}$ and cannot be transmitted through the SNP effects.

Correlations for the last 2 groups of young animals were lower than for the other groups and reduced the overall correlations as well. To recover the missing information in the SNP model, we applied 2 adjustments to the GEBV of animals from these 2 groups:

- In the case of a dam with phenotype but no genotype in the previous run, we estimated her Mendelian sampling term $\hat{\mathbf{m}}$ and the reliability $\left(r^{2}\right)$ of the imputation of her genotype $\hat{r}_{i m p}^{2}$ and added the term $0.5 \hat{\mathbf{m}}\left(1-\hat{r}_{i m p}^{2}\right)$ to the GEBV of the young animal. Weighting by this function of the imputation reliability should account for the implicit imputation that takes place in single-step evaluations (Fernando et al., 2014). For instance, dams with several offspring in the system have their genotype relatively well imputed and contribute to the estimation almost as if they were genotyped. Their correction term should accordingly receive a lower weight. The program ApaX99 (MiX99 Development Team, 2016) was used to approximate $\hat{r}_{i m p}^{2}$, assuming an animal model for predicting the gene contents of ungenotyped animals from genotype records on their relatives (Gengler et al., 2007).

- In the case of an unknown dam, we identified which genetic group would replace her in the pedigree of the full model, and half of the solution of this genetic group in the April 2017 run was added to the GEBV of the young animal.

Results from the SNP model and these 2 adjustments are also presented in Table 1 and referred to as the "SNP model PLUS." These results were obtained with the PA approximation, mostly with 3 sire PA. Correlations from this model averaged 0.9932 (from 0.990 to 0.995 across traits), slightly higher than those from the $\mathrm{G}$ model. Of course, these adjustments could also have been applied to solutions from the G model, which would have improved its correlations as well. We intentionally focused on the results from the SNP model, for reasons that will be discussed later.

Figure 1 depicts the effects of the 2 applied adjustments on the correlations of the 2 groups and on the overall correlation. Results are presented for rump angle as an illustration, but the same effects were observed for all other traits. The effects of these adjustments make clear that the imprecision in the GEBV from the alternative models did not necessarily come from an inability of the models to efficiently transmit the information from the predictor to the predictand. Rather, this imprecision came from some piece of information that was not contained in the vector of observations, $\hat{\mathbf{a}}_{2}$, at all and therefore could not be corrected through an improvement in the estimation method. In fact, correlations from both the G model and the SNP model were quite high. Improvements could only be achieved by recovering information from outside the set of genotyped animals.

These results may also contribute to ongoing research on the development of methods to approximate reliabilities of single-step breeding values. For instance, the method proposed by Liu et al. (2017) to harmonize calculation of genomic reliabilities among Interbull members relies on prediction error variances of SNP effects. If prediction error variances of SNP effects are derived only from genotyped individuals, the Mendelian sampling contribution of an ungenotyped dam with phenotype to the reliability of the breeding value of a new candidate may need to be considered afterward. Whenever good estimates of single-step reliabilities are available, an improvement of the indirect predictions could be attempted by using them to derive proper weights in the model.

The $\mathrm{G}$ model requires setting up, inverting, and storing the inverted $\mathbf{G}$ matrix, which could become impractical with a steady increase in the number of genotyped animals. Misztal et al. (2014) proposed an alternative way of computing $\mathbf{G}^{-1}$ with the algorithm for proven and young (APY), in which the matrix to be inverted contains only a subset of the genotyped animals, the so-called core animals. Lourenco et al. (2018) reported results with a $\mathbf{G}^{-1}$ constructed using APY that were close enough to the results using the regular $\mathbf{G}^{-1}$. Nevertheless, they pointed out that the matrix $\mathbf{G}^{-1}$ to be stored on disk for future indirect predictions was still of considerable size due to relationships between core and noncore animals. To solve this problem, they suggested an additional approximation by using a block of the matrix $\mathbf{G}^{-1}$ containing only the core animals for the 

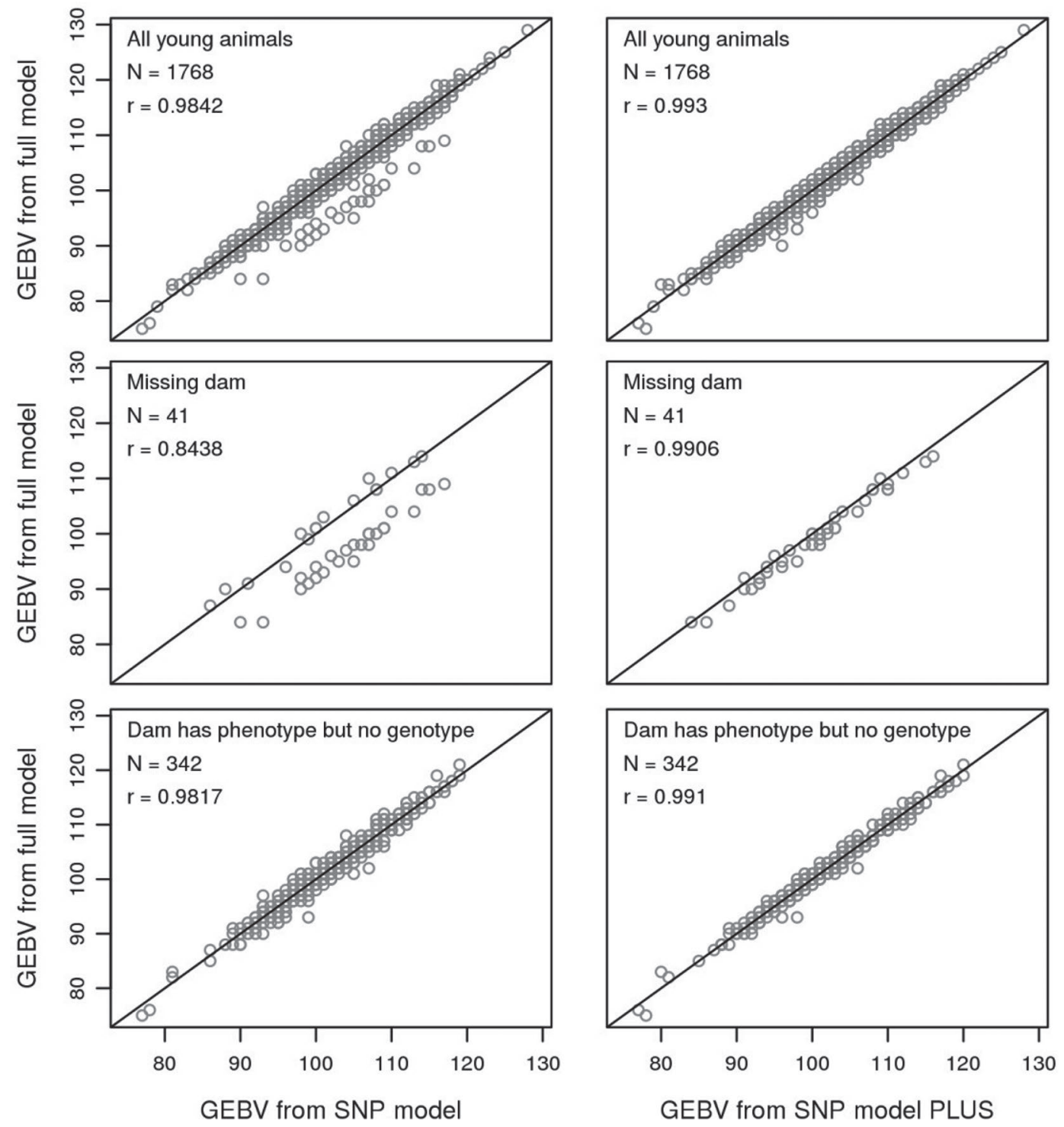

Figure 1. Genomic breeding values (GEBV) for rump angle from the full model against the GEBV from the SNP model before and after adjustments (PLUS) for different groups of young animals.

indirect prediction, but this decreased the correlations between indirect predictions and GEBV from the full model.

In the classical implementation of single-step, with the explicit computation of the $\mathbf{H}^{-1}$ block $\left[\mathbf{G}^{-1}-\mathbf{A}_{22}^{-1}\right]$, the matrix $\mathbf{G}^{-1}$ has to be computed anyway and can be used afterward for the prediction of future young animals with the $\mathrm{G}$ model. This classical approach might not be the best method for systems with hundreds of thousands of genotyped animals, however, which is already a real scenario in some breeding programs. Alternative approaches that avoid the explicit calculation of the matrix $\mathbf{G}^{-1}$ are under development, such as the ssGTBLUP method (Mäntysaari et al., 2017). In such cases, the SNP model seems to be the obvious solution for the interim prediction of GEBV for young animals without running the full single-step evaluation again.

The major advantage of the SNP model over the G model is that the coefficient matrix to be inverted or decomposed when solving the equations is of equal size to the number of markers, which is often lower than the number of genotyped animals and should remain constant as long as chips of similar density are used. If the transmission of the residual polygenic information is approximated with PA calculated from $\hat{\mathbf{e}}$, then all data needed for prediction of young candidates can be stored in the vectors $\hat{\mathbf{g}}$ and $\hat{\mathbf{e}}$. This method is very inexpensive and can be easily implemented on a database level, al- 
lowing for instantaneous predictions. Note that replacing $\mathbf{C}$ by $\mathbf{M}^{\prime}$ in the $\mathrm{G}$ model would also yield SNP effects but still requiring $\mathbf{G}^{-1}$, which one may wish to avoid. As mentioned before, the residual term $\hat{\mathbf{e}}$ may contain some estimation error other than that associated with a polygenic effect. Some way of accounting for that should be the subject of further research.

In conclusion, prediction of single-step breeding values for young animals can be well approximated using SNP effects estimated from systems of size equal to the number of markers. The SNP model PLUS presented here can be used to predict GEBV of young selection candidates in a very cost-effective manner and is well suited for interim evaluations in short time intervals or even on a daily basis.

\section{ACKNOWLEDGMENTS}

We gratefully acknowledge the Arbeitsgemeinschaft Süddeutscher Rinderzucht- und Besamungsorganisationen e.V. (Grub, Germany) for their financial support within the research cooperation "Zukunftswege." We thank two anonymous reviewers for useful comments and suggestions.

\section{REFERENCES}

Christensen, O. F., and M. S. Lund. 2010. Genomic prediction when some animals are not genotyped. Genet. Sel. Evol. 42:2.

Edel, C., H. Schwarzenbacher, H. Hamann, S. Neuner, R. Emmerling, and K.-U. Götz. 2011. The German-Austrian genomic evaluation system for Fleckvieh (Simmental) cattle. Interbull Bull. 44:152156.

Fernando, R. L., J. C. M. Dekkers, and D. J. Garrick. 2014. A class of Bayesian methods to combine large numbers of genotyped and non-genotyped animals for whole-genome analyses. Genet. Sel. Evol. 46:50.

Gengler, N., P. Mayeres, and M. Szydlowski. 2007. A simple method to approximate gene content in large pedigree populations: application to the myostatin gene in dual-purpose Belgian Blue cattle. Animal 1:21-28.

Henderson, C. R. 1985. Best linear unbiased prediction of nonadditive genetic merits in noninbred populations. J. Anim. Sci. 60:111-117.
Legarra, A., I. Aguilar, and I. Misztal. 2009. A relationship matrix including full pedigree and genomic information. J. Dairy Sci. 92:4656-4663.

Liu, Z., P. M. VanRaden, M. H. Lidauer, M. P. Calus, H. Benhajali, H. Jorjani, and V. Ducrocq. 2017. Approximating genomic reliabilities for national genomic evaluation. Interbull Bull. 51:75-85.

Lourenco, D. A. L., S. Tsuruta, B. O. Fragomeni, Y. Masuda, I. Aguilar, A. Legarra, J. K. Bertrand, T. S. Amen, L. Wang, D. W. Moser, and I. Misztal. 2015. Genetic evaluation using single-step genomic best linear unbiased predictor in American Angus. J. Anim. Sci. 93:2653-2662.

Lourenco, D. A. L., S. Tsuruta, B. O. Fragomeni, Y. Masuda, I. Aguilar, A. Legarra, S. Miller, D. Moser, and I. Misztal. 2018. Single-step genomic BLUP for national beef cattle evaluation in US: From initial developments to final implementation. Page 495 in Proc. 11th World Congr. Genetics Appl. Livest. Prod., Auckland, New Zealand. http://www.wcgalp.org/proceedings/2018/ single-step-genomic-blup-national-beef-cattle-evaluation-us-initial -developments.

Mäntysaari, E. A., R. D. Evans, and I. Strandén. 2017. Efficient singlestep genomic evaluation for a multibreed beef cattle population having many genotyped animals. J. Anim. Sci. 95:4728-4737.

MiX99 Development Team. 2016. MiX99: A software package for solving large mixed model equations. Release XI/2016. Natural Resources Institute Finland (Luke), Jokioinen, Finland.

Misztal, I., A. Legarra, and I. Aguilar. 2014. Using recursion to compute the inverse of the genomic relationship matrix. J. Dairy Sci. 97:3943-3952.

Misztal, I., Z. G. Vitezica, A. Legarra, I. Aguilar, and A. A. Swan. 2013. Unknown-parent groups in single-step genomic evaluation. J. Anim. Breed. Genet. 130:252-258.

Patry, C., and V. Ducrocq. 2011. Accounting for genomic pre-selection in national BLUP evaluations in dairy cattle. Genet. Sel. Evol. 43:30.

Shabalina, T., E. C. G. Pimentel, C. Edel, L. Plieschke, R. Emmerling, and K.-U. Götz. 2017. Short communication: The role of genotypes from animals without phenotypes in single-step genomic evaluations. J. Dairy Sci. 100:8277-8281.

Strandén, I., and K. Vuori. 2006. RelaX2: Pedigree analysis program. Pages 27-30 in Proc. 8th World Congr. Genet. Appl. Livest. Prod., Belo Horizonte, MG, Brazil. http://www.wcgalp.org/proceedings/ 2006/relax2-pedigree-analysis-programme.

VanRaden, P. M. 2008. Efficient methods to compute genomic predictions. J. Dairy Sci. 91:4414-4423.

Wang, H., I. Misztal, I. Aguilar, A. Legarra, and W. M. Muir. 2012. Genome-wide association mapping including phenotypes from relatives without genotypes. Genet. Res. 94:73-83.

Westell, R. A., R. L. Quaas, and L. D. Van Vleck. 1988. Genetic groups in an animal model. J. Dairy Sci. 71:1310-1318.

Wiggans, G. R., P. M. VanRaden, and T. A. Cooper. 2015. Technical note: Rapid calculation of genomic evaluations for new animals. J. Dairy Sci. 98:2039-2042. 